\title{
Implementing a peer support programme in a regional mental health service: A personal journey
}

\author{
Gabrielle Vilic, Chris Lloyd, Samson Tse
}

Peer support is a well established component of many mental health services that provides a promising employment opportunity and recognises experience with mental illness as an asset rather than a liability. The overall aim of this paper is to discuss the challenges of implementing a peer support programme in a regional mental health service. In particular, the article discusses how the roles and functions of peer support workers have evolved and how challenges to service effectiveness were identified and overcome. The authors also examine the development of a related consumer companion role and consider the similarities and differences of peer support and consumer companion roles. Recommendations are made with respect to issues such as how allied health professionals views this role, what happens when peer support workers become ill and level of training and supervision.

Key words: Peer support $\quad$ Peer support worker Consumer companion Mental health

Submitted 29 May 2015; sent back for revisions 23 June 2015; accepted for publication following double-blind peer review 31 September 2015

Gabrielle Vilic,

Former consumer consultant, Gold Coast Health Service District, Queensland, Australia and currently Director

of Social Inclusion and Recovery, Metro South Addictions and Mental Health Service, Queensland, Australia; Chris Lloyd,

Senior research fellow, Behavioural Basis of Health, Griffith University, Queensland, Australia; Samson Tse,

Professor, Department of Social Work and Social Administration,

The University of Hong

Kong, Hong Kong.

Correspondence to: Gabrielle Vilic E-mail:

Gabrielle.Vilic@health. qld.gov.au
$\mathrm{P}$ eer support is social emotional support, frequently coupled with instrumental support, that is provided by people with a mental illness to others sharing a similar mental health condition in their recovery journey (Solomon, 2004; Ockwell, 2012; Johnson et al, 2014). The shift to a recovery paradigm has helped to drive service providers towards employing service users who have recovered as peer support workers to support and assist current patients and service users in their recovery journey (Wallcraft, 2012).

Moll et al (2009) suggest that peer support provides a promising employment opportunity that recognises experience with mental illness and the mental health system as an asset rather than a liability. They claim that peer support represents meaningful work for mental health consumers and that it benefits staff, consumers and the agencies that employ them.

Peer support worker positions are a valuable employment opportunity for people with mental illness and is an emerging workforce in the mental health system (Tse et al, 2014). The employment of people with mental illness as providers may be a critical pathway for increasing consumer involvement in the mental health service system (Hutchinson et al, 2006; Simpson et al, 2014). It has been stated that this would lead to a transformation of a mental health system where service delivery would no longer be driven solely by the values of professionals but rather be based upon an equitable partnership involving individuals with mental illness (Tse et al, 2012; Slade et al, 2014). Kemp and Henderson (2012) suggest that for this to happen, and so as not to lose an otherwise valuable resource to the mental health system, there is a need for the provision of training for managers and inclusion of the peer support worker role in orientation programmes.

This paper aims to discuss the challenges experienced by the consumer consultant when implementing a peer support programme in a regional mental health service.

\section{LITERATURE REVIEW}

Peer support has a long tradition in informal services and may complement mental health care in promoting recovery orientation and destigmatisation. Mahlke et al (2014) identified a number of crucial issues for implementation, which may include the clarification of role and job descriptions of peer support workers and non-peer staff, organisational support strategies, training and adequate supervision. Peer support workers are increasingly employed by mental health services in the UK, US, Sweden, New Zealand and Australia in a wide range of roles, 
services and settings (Moran et al, 2013; Landers and Zhou, 2011). Examples include:

Working with psychiatric inpatients (Bouchard et al, 2010)

- Facilitating recovery groups for veterans (Beehler et al, 2014)

Conducting peer support groups in inpatient settings (Sledge et al, 2011) and the community (Stant et al, 2011)

Working alongside mental health professionals while conducting case management (Rivera et al, 2007)

Being involved in research and evaluation (Mayes, 2010; Kara, 2013).

Peer support programmes have been established internationally and have been appraised in qualitative literature as being beneficial for service users. However, in a systematic review conducted by Lloyd-Evans et al (2014), they found little evidence from current trials about the effects of peer support for people with severe mental illness. They suggested that if peer support programmes are identified as desirable and implemented locally, service planners should include rigorous evaluation to determine whether these programmes affect outcomes of interest.

Chinman et al (2014) conducted a review to assess the level of evidence and effectiveness of peer support services delivered by individuals in recovery to those with serious mental illness. Although the level of evidence was moderate across all service types (peers added to traditional services, peers in existing roles and peers delivering curricula), improvements were to be seen in the following outcomes: reduced inpatient services; improved relationships with providers; better engagement with care; higher levels of empowerment; higher levels of patient activation; and higher levels of hopefulness for recovery.

\section{Peer support in facilitating recovery}

Connecting with others who have a mental illness is seen as facilitating recovery (Schon, 2010). Consumer-operated services in the form of peer support were found to be positively associated with many indices of recovery and empowerment in a study conducted by Corrigan (2006). Resnick and Rosenheck (2008) compared the effectiveness of a peer education and support programme based on the measures of recovery orientation, confidence and empowerment. Findings revealed that participants in peer support were associated with enhanced personal wellbeing, as measured by recovery-oriented and more traditional clinical measures.

The aim of the study conducted by Happell (2008) was to explore service user perspectives in relation to factors that promote and impede recovery. Two main themes arose during the data analysis process. These were 'treatment' and 'support and social connectedness'. Medication was viewed as an important part of treatment and a number of non-pharmaceutical interventions that were also considered to be useful were mediation, counselling, and having a crisis management plan. Support from both staff and peers emerged as the more important and influential factor.

Peer support is built on the premise that people who have faced, endured and overcome mental illness can offer useful support, hope and encouragement to others facing similar problems (Schon, 2010). The study explored how individuals with experience of severe mental illness in Sweden perceived peer support in facilitating their recovery. The results show the importance of meeting others in recovery to experience the power of identification, where participants described how peer support meant an end to isolation and became an arena for identification, normalisation, connection, and being important to others.

Schon (2011) suggested that peer support programmes have an important impact on facilitating recovery processes and provide a base for further growth, as well as the possibility for people in recovery to participate in all aspects of community, such as having a job. However, Lloyd-Evans et al (2014) found in their metaanalysis that no studies reported employment as a significant outcome.

\section{Challenges experienced by peer support workers}

Moran et al (2013) explored the challenges experienced by 31 peer providers in diverse settings and roles using in-depth interviews. Challenges included the lack of clear job descriptions; lack of skills for using one's life story and lived experience; lack of helping skills; and negative aspects of carrying a peer provider label. The authors concluded that it is advised to address peer identity and self-definition issues early in training. It was stated that the challenge of practising self-care and the importance of being informed about job accommodations and rights is an integral part of peer work in the face of possible risks for excessive emotional labour and recurrence of symptoms.

Kemp and Henderson (2012) used the nominal group technique to collect and analyse the data from a study of peer support workers in Western Australia. The main challenge encountered by participants was a lack of understanding of the 
peer support role, which caused them to feel a sense of exclusion. It was strongly felt that managers have a responsibility to be informed about the peer support worker role and communicate role-related information to other team members to ensure that peer support workers are included as part of the health care team.

In New Zealand, Scott and Doughty (2012) conducted a qualitative study based on two individual or group interviews with each of 37 peer supporters and peer support managers. The findings revealed that, generally, peer supporters conceptualise their practice within a discourse of empowerment and strength-based philosophy. A major issue that was identified was documentation, and in response to this, peer support workers developed a method of notetaking in which they wrote from a peer support perspective with an emphasis on hopefulness.

Johnson et al (2014) felt that the potential benefits to peer support workers themselves was not well understood and so conducted a survey of peer support workers to examine potential benefits. A variety of benefits was reported, including greater confidence as workers and improved perceptions of their own health and recovery. There were also a small number of negative comments, almost exclusively about the low salary and benefits. Many of the respondents had been unemployed for a long time before finding employment as a peer support worker. The authors thus concluded that employment may be a potent vehicle for improving recovery, interpersonal relations, quality of life and career development in peer support workers.

\section{CASE EXAMPLE}

The following section describes the first author's experience of developing a consumer workforce in a regional health district from the perspective of having a lived experience. To date, this programme has not been formally evaluated.

\section{Consumer consultant}

In 2005, the Gold Coast Health Service District employed one full-time consumer consultant. This was the introduction to a consumer role to the district. Responsibilities included:

Communicating with consumers, carers, professional groups and all service providers to assist in planning, delivering and evaluating a mental health consumer-focused service

Building and maintaining effective relationships and networks with community support and advocacy providers
Providing assistance to the service

Actively investigating and identifying areas for improvement to service, policy and procedures from a mental health service consumers' perspective.

During my first year as a consumer consultant, I felt disempowered and sometimes experienced a sense of shame when some of the staff who had previously treated me completely ignored me (even though this admission was 11 years ago). My experience is reflected in Nestor and Galletly (2008), who found that peer support workers experience discrimination from staff with respect to their ability to work. Despite the difficulties experienced, I decided that the best thing to do was to concentrate on what could be achieved and to focus on that. This approach helped me to gain strength, especially when I could see that consumers were benefiting from the services that I was able to provide.

On starting my new role, I got involved in the formation of consumer-facilitated groups, one of which was the Issues and Concerns Group. Some of the issues raised pertained to the environment, maintenance and treatment, while others centred on staff. It was fortunate that the services I provided were closely allied with the rehabilitation service, and the staff working in that service provided me with the support I needed. After discussion about the negative interpretation of the role, it was decided that the role needed to appear less intimidating. It was thought important that the consumer consultant be able to actively work with staff to initiate change, rather than be seen as working against them. As a result, the Issues and Concerns Group was changed to a one-to-one request from the consumer to the consumer consultant.

Fortnightly supervision was provided by the manager, and bi-monthly supervision by the executive director. This was vital to the development of the rolet. Peer supervision was also available from the senior project officer in the Mental Health Directorate. It was important to have supervision and support from outside the district as it provided impartial feedback.

\section{Peer support workers}

When funding became available, two peer support workers were hired. The need to employ peer/ recovery support workers who could continue to work on the inpatient wards and support the consumer consultant to work more strategically had been identified. This was critical in being able to expand the service and also to give me the support of other peer support workers while continuing to develop the service. 
The peer support workers had not received training previously, which was then provided by the mental health service. A large part of the role comprises providing hope and empowering consumers on the ward, being able to develop rapport and providing a different perspective to the clinician. Groups that have been facilitated by the peer support workers include Hope and Recovery, Managing Your Illness and Medication, Lifestyle, Mental Health Tribunal Group, and the Pastoral Care Group.

The consumer consultant and peer support workers played a pivotal role in developing groups and focusing on the consumer perspective in the inpatient wards. The Hope and Recovery Group have been very successful and is now facilitated twice per week. This group provides a friendly approach to mental health issues and emphasises and supports each individual's potential for recovery. The peer support workers reported that their roles assimilated in the inpatient wards with few issues.

Over time, a shift in staff attitudes has been observed. It would appear that the staff now recognise the value of the consumer workers and identify the rapport that the consumer workers have with patients due to their lived experience of mental illness. The peer support workers are able to write clinical notes, use the electronic patient record and attend handover meetings. These are essential components of work on the inpatient unit and it has taken years to achieve this level of participation.

With service development, it was decided that the peer support roles be integrated into the community clinics to provide transition for service users from inpatient to the community. This has been challenging at times for the peer support workers, as the community have not previously had much involvement with consumer participation. The transition has been challenging, from working in a wellsupported team (psychiatric rehabilitation) to an environment where the peer support workers had not previously worked. Additionally, the change from an inpatient to a community environment involved making large changes to work practices.

The peer support workers were supported through supervision during this period and receive fortnightly supervision from the consumer consultant. During supervision, it is important to provide support not only in terms of work practice but also emotionally. There is also supervision available by telephone from the consumer peer supervisor from the Directorate. There have been no issues to date with the peer support workers becoming unwell.

\section{Consumer companions}

Three years ago, the Gold Coast became one of the pilot sites for the Consumer Companion Programme. The programme was developed due to an increase in feelings of loneliness, boredom and lack of support reported by inpatients. Consumer companions are people who have a lived experience of mental illness and provide interaction and support for consumers in acute inpatient units or extended care facilities.

Seven consumer companions were hired and they work 3-hour shifts 2-3 times per week. They are not responsible for facilitating any peer support groups. Rather, they work on an individual basis and provide support to consumers on the inpatient wards. The role of the consumer companion involves:

Providing and engaging in activities

Sharing their lived experience with consumers

Acting as a positive role model and promoting hope and recovery

Showing empathy and understanding

Providing companionship and guidance on the wards and for life outside the hospital.

The consumer companions play a pivotal role in supporting other consumers with mental illness. They take part in everyday activities at the ward and mingle with inpatient service users. Conversations are goal-driven in that they are usually around a shared recovery agenda.

Monthly group supervision is provided by the programme supervisor and consumer consultant to address communication, strategies, role-plays, scenarios with consumers on the wards, code of conduct, assertiveness and de-escalation.

Working as a peer support worker fosters recovery and enhances the humanistic growth of the individual by supporting and caring for others (Moran et al, 2012). Simpson et al (2014) described the preparation, selection, training and support of people with lived experience of mental illness working as peer support workers. The trainees' feedback suggested that the training was a valuable and challenging, yet positive experience that provided them with good preparation for the role. A key area they identified they could have been better prepared for concerned the emotional involvement and feelings they would have for their peers and in particular in regard to ending the relationship.

Given that it has been suggested that training is critical for peer support workers (Chinman et al, 2008; Simpson et al, 2014), a training programme was introduced for all consumer employees. It is compulsory for all new consumer employees of the District to attend sessions or workshops on code of conduct, 


\section{KEY POINTS}

- Peer support has a long tradition in promoting recovery orientation in mental health and is an emerging workforce in the mental health system

- Peer support workers communicate with consumers, carers, professional groups and service providers to assist in planning, delivering and evaluating a mental health consumer-focused service

- Peer support workers face many challenges when working in mental health services, including discrimination from staff with respect to their ability to work.

orientation, Aboriginal and Torres Strait Islander cultural awareness, emergency procedures, and aggression behaviour management.

Many members of the consumer workforce are undertaking further study in mental health at the local community college. This has been a good opportunity for the team of consumer workers to increase their knowledge, up skill and gain confidence in themselves and their roles. As noted by Cleary et al (2006), receiving adequate training and education is a pressing issue for peer support workers. The peer support workers in this programme have been able to access both mandatory training (e.g. code of conduct, crisis interventions) and selective training (e.g. working with families), which has greatly assisted them in providing the service.

\section{CONCLUSIONS}

This paper described the process of implementing a peer support programme in a regional mental health service and the issues and challenges that need to be overcome in order to expand the consumer workforce. In the time that the District has employed consumers, the consumer programme has continued to expand, and it is hoped that this programme will continue to grow due to its success.

\section{Conflict of interest: None declared.}

Beehler S, Clark JA, Eisen SV (2014) Participant experiences in peer- and clinician-facilitated mental health recovery groups for veterans. Psychiatr Rehabil J 37(1): 43-50

Bouchard L, Montreuil M, Gros C (2010) Peer support among inpatients in an adult mental health setting. Issues Ment Health Nurs 31(9): 589-98

Chinman M, Young AS, Hassell J, Davidson L (2006) Toward the implementation of mental health consumer provider services. J Behav Health Serv Res 33(2): 176-95

Chinman M, Lucksted A, Gresen R et al (2008) Early experiences of employing consumer-providers in the VA. Psychiatr Serv 59(11): 1315-21

Chinman M, George P, Dougherty RH et al (2014) Peer support services for individuals with serious mental illnesses: Assessing the evidence. Psychiatr Serv 65(4): 429-41

Cleary M, Walter G, Escott P (2006) 'Consumer consultant': Expanding the role of consumers in modern mental health services. Int J Ment Health Nurs 15(1): 29-34
Corrigan PW (2006) Impact of consumer-operated services on empowerment and recovery of people with psychiatric disabilities. Psychiatr Serv 57(10): 1493-6

Happell B (2008) Determining the effectiveness of mental health services from a consumer perspective: Part 1 . Int Ment Health Nurs 17(2): 116-22

Hutchinson DS, Anthony WA, Ashcraft L et al (2006) The personal and vocational impact of training and employing people with psychiatric disabilities as providers. Psychiatr Rehabil J 29(3): 205-13

Johnson G, Magee C, Maru M, Furlong-Norman K, Rogers ES, Thompson K (2014) Personal and societal benefits of providing peer support. Psychiatr Serv 65(5): 678-80

Kara H (2013) Mental health service user involvement in research. Journal of Public Mental Health 12(3): 122-35

Kemp V, Henderson AR (2012) Challenges faced by mental health peer support workers: Peer support from the peer supporter's point of view. Psychiatr Rehabil J 35(4): 337-40

Landers GM, Zhou M (2011) An analysis of relationships among peer support, psychiatric hospitalization, and crisis stabilization. Community Ment Health J 47(1): 106-12

Lloyd-Evans B, Mayo-Wilson E, Harrison B et al (2014) A systematic review and meta-analysis of randomised controlled trials of peer support for people with severe mental illness. BMC Psychiatry 14: 39

Mahlke CI, Kramer UM, Becker T, Bock T (2014) Peer support in mental health services. Current Opinion in Psychiatry 27(4): 276-81

Mayes D (2010) When it pays to be bipolar. Mental Health and Social Inclusion 14(1): 20-3

Moran GS, Russinova Z, Stepas K (2012) Toward understanding the impact of occupational characteristics on the recovery and growth processes of peer providers. Psychiatr Rehabil J 35(5): 376-80. doi: 10.1037/h0094496

Moran GS, Russinova Z, Gidugu V, Gagne C (2013) Challenges experienced by paid peer providers in menta health recovery Community Ment Health J 49(3): 281-91

Moll S, Holmes J, Geronimo J, Sherman D (2009) Work transitions for peer support providers in traditional mental health programs. Work 33(4): 449-58

Nestor P, Galletly C (2008) The employment of consumers in mental health services. Australas Psychiatry 16(5): 344-7

Ockwell C (2012) Challenges and triumphs: Developing an inpatient peer support project. The Journal of Mental Health Training, Education and Practice 7(2): 94-100

Resnick SG, Rosenheck RA (2008) Integrating peer-provided services: A quasi-experimental study of recovery orientation, confidence, and empowerment. Psychiatr Serv 59(11): 1307-14. doi: 10.1176/appi.ps.59.11.1307

Rivera JJ, Sullivan AM, Valenti SS (2007) Adding consumerproviders to intensive case management: Does it improve outcome? Psychiatr Serv 58(6): 802-9

Schon U (2010) The power of identification: Peer support in recovery from mental illness. Scandinavian Journal of Disability Research 12(2): 83-90

Scott A, Doughty C (2012) "Confronted with paperwork" Information and documentation in peer support. J Ment Health 21(2): 154-64

Simpson A, Quigley J, Henry SJ, Hall C (2014) Evaluating the selection, training, and support of peer support workers in the United Kingdom. J Psychosoc Nurs Ment Health Serv 52(1): 31-40. doi: 10.3928/02793695-20131126-03

Slade M, Amering M, Farkas M et al (2014) Uses and abuses of recovery: Implementing recovery-oriented practices in mental health systems. World Psychiatry 13(1): 12-20

Sledge WH, Lawless M, Sells D, Wieland M, O'Connell MJ, Davidson L (2011) Effectiveness of peer support in reducing readmissions of persons with multiple psychiatric hospitalizations. Psychiatr Serv 62(5): 541-4

Solomon P (2004) Peer support/peer provided services underlying processes, benefits, and critical ingredients. Psychiatr Rehabil J 27(4): 392-401

Stant AD, Castelein S, Bruggeman R et al (2011) Economic aspects of peer support groups for psychosis. Community Ment Health J 47(1): 99-105

Tse S, Cheung E, Kan A, Ng R, Yau S (2012) Recovery in Hong Kong: Service user participation in mental health services. Int Rev Psychiatry 24(1): 40-7

Tse S, Tsoi EW, Wong S, Kan A, Kwok CF (2014) Training of mental health peer support workers in a non-western high-income city. Int J Soc Psychiatry 60(3): 211-8

Wallcraft J (2012) What has been learned from joint working between mental health professionals, patients and users of psychiatric services, their families and friends? Curr Opin Psychiatry 25(4): 317-21 